\title{
Clinical
}

\section{Hepatic Angiomyolipoma Mimicking Malignancy: A Case Report}

\author{
Kazuyoshi Suzuki ${ }^{1)}$, Takeo Mazaki ${ }^{1)}$, Jun Mannmoto ${ }^{1)}$, Yukimoto Ishi $^{1)}$, Hideki Masuda ${ }^{1)}$, \\ Akihiro HENNMI ${ }^{2)}$ and Tadatoshi TAKAYAMA ${ }^{1)}$ \\ ${ }^{1)}$ Department of Digestive Surgery, and ${ }^{2}$ Department of Pathology, Nihon University School of Medicine
}

\begin{abstract}
Hepatic angiomyolipoma (AML) is an uncommon benign mesenchymal tumor of the liver. Most AMLs of the liver are asymptomatic and are discovered incidentally, but the diagnosis remains difficult. We report a case of a 33-year-old woman who had an abdominal mass and in whom diagnosis remained unidentified after imaging and needle biopsy. On dynamic CT, a heterogeneous enhanced hepatic lesion was revealed in the arterial phase with washout in the portal phase. On MRI, it was shown as a hypointense tumor on T1 weighted images and hyperintense on T2 weighted images. Because hepatocellular carcinoma was suspected as a result of the needle biopsy, hepatic segmentectomy was performed. The final pathological diagnosis was AML due to positive HMB45. AML needs to be considered as a condition that may display the atypical images consistent with liver tumor. In cases such as this, we should request the examination of immunohistochemical staining especially for HMB45 on biopsy specimens.
\end{abstract}

Key wards: liver tumor, angiomyolipoma, hepatic resection, HMB45

(J. Nihon Univ. Med. Ass., 2008; 67 (4): 238-241)

\section{Introduction}

Angiomyolipoma (AML) is a rare mesenchymal tumor that occurs most commonly in the kidney, and is occasionally found in the liver. The patient usually has an asymptomatic course and the tumor is often incidentally revealed. Histologically, AML is characterized by a mixture of mature fat cells, blood vessels and smooth muscle cells in various proportions ${ }^{1)}$. The imaging features of AML depend on the relative proportions of tissue components within the tumor ${ }^{2}$. Although the radiological, histological and immunochemical features are described in the literature, preoperative correct diagnosis of AML is occasionally difficult $t^{3)}$, and is often misdiagnosed as hepatocellular carcinoma $(\mathrm{HCC})^{4}$. Immunoreactivity for actin, desmin, s-100 protein, and HMB45 is a good indicator for determining the final diagnosis of $\mathrm{AML}^{5}$.

We described a case of AML for which a definitive diagnosis could not be made on images and needle biopsy preoperatively.

\section{Case report}

A 33-year-old woman was admitted due to abdominal pain in the right upper quadrant. Physical examination showed right upper portion of abdominal tenderness without rebound tenderness, no jaundice, and no significant medical history. She has a history of excessive alcohol consumption (two bottles of clear liquor a day for 9 years). A liver function test revealed AST 74 U/L, ALT 34 U/L and $\gamma$-GTP 264 U/L. Hepatitis B and $\mathrm{C}$ virus were negative. The plasma levels of AFP and PIVKA-II were within normal limits.

Ultrasound study (US) showed a well-defined hypoechoic mass mixed with small hyperechoic areas in the posterior inferior portion (segment 6) of the liver (Fig. 1). Contrast enhanced dynamic computed tomography (CT) revealed an inhomogeneous enhanced lesion in the arterial phase with washout in the portal phase (Fig. 2). On MRI the lesion was hypointense on T1 weighted magnetic resonance images (MRI), and hyperintense on T2 and heavy T2 weighted images. On gadolinium enhanced dynamic MRI, the lesion showed early enhancement but the central portion was not enhanced in the arterial phase. In the portal phase, wash-out of contrast material was observed (Fig. 3). According to those observations, the differential diagnoses were considered as follows: HCC, focal nodular hyperplasia $(\mathrm{FNH})$ and hepatic adenoma. Ultrasound guided needle biopsy was performed and HCC was suspected pathologically.

The patient underwent resection of segment- 6 . The tumor was $5.5 \mathrm{~cm} \times 4.5 \mathrm{~cm} \times 4.5 \mathrm{~cm}$ in size. The cut surface of the tumor was nodular, yellow to tan and a 


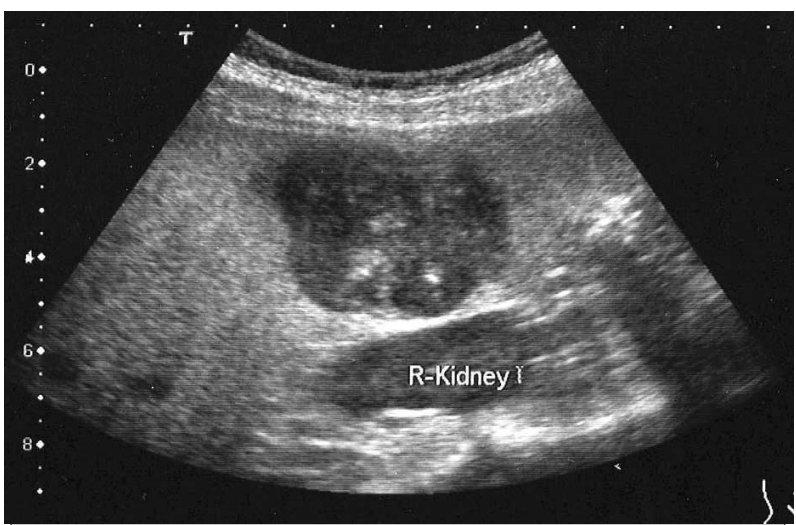

Fig. 1 Ultrasonography showing a well-defined hypoechoic mass with small hyperechoic component in segment 6 .

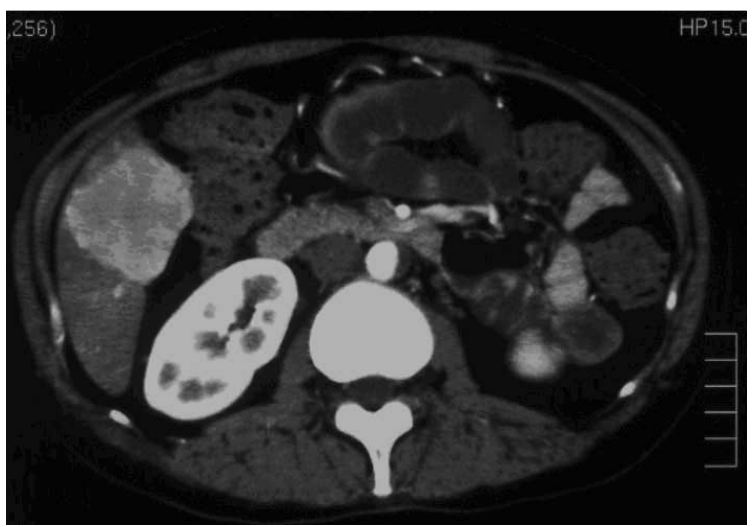

A

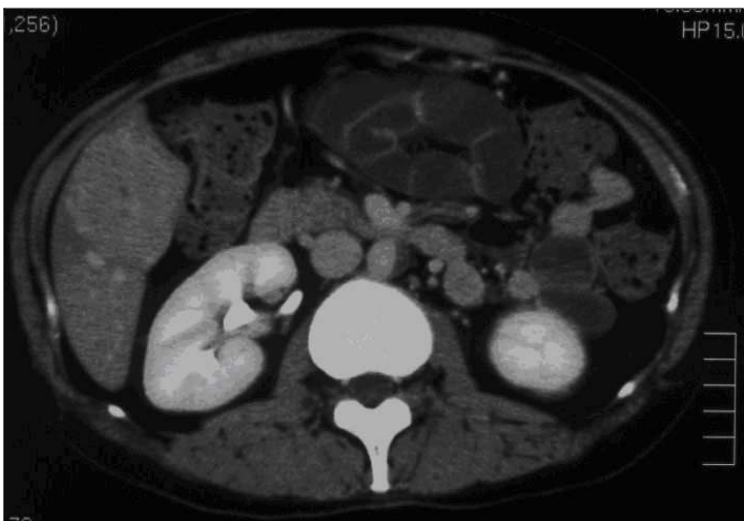

B

Fig. 2 Computed tomography revealing an irregular shaped inhomogeneous enhanced lesion in the arterial phase (A) and wash-out in the portal phase (B).

well-defined margin with no capsule (Fig. 4). The tumor surgically removed was composed of irregularly arranged sheets and interlacing bundles of epithelioid smooth muscle cells and a small amount of mature adipose tissue (Fig. 5). Thick-walled, medium-sized vessels were also observed as the tumor elements. The epithelioid smooth muscle cells had eosinophilic or

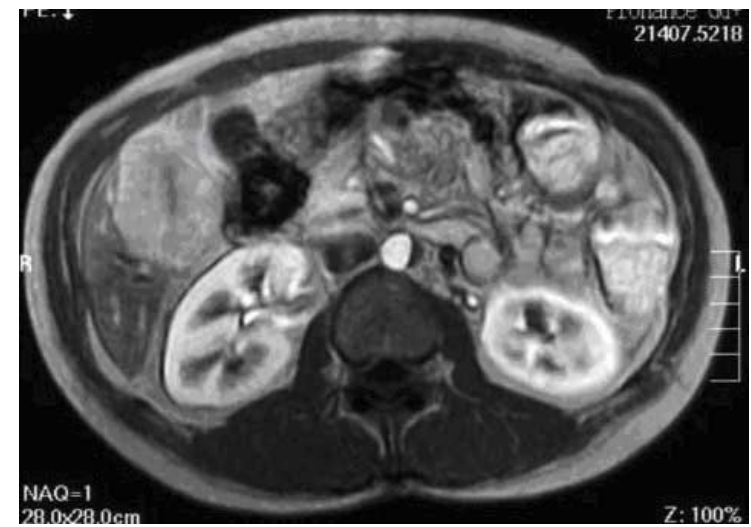

A

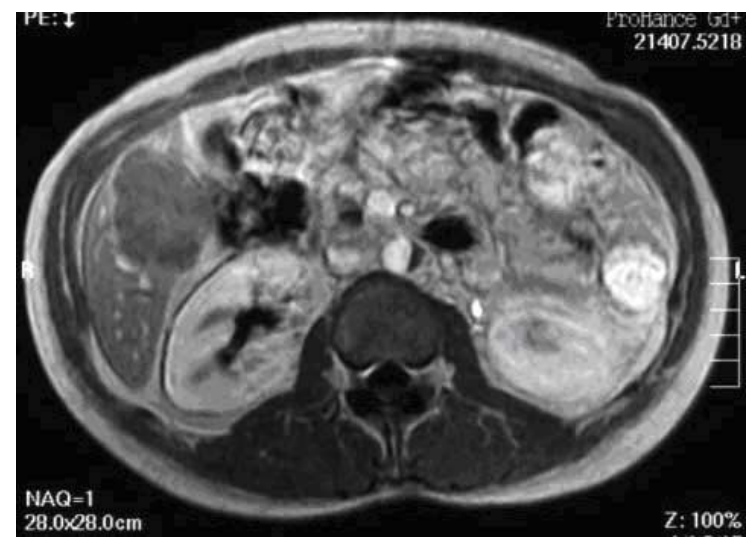

B

Fig. 3 MRI revealing the lesion shows early enhancement but the central portion is not enhanced in the arterial phase (A), wash-out of contrast material is observed in the portal phase (B).

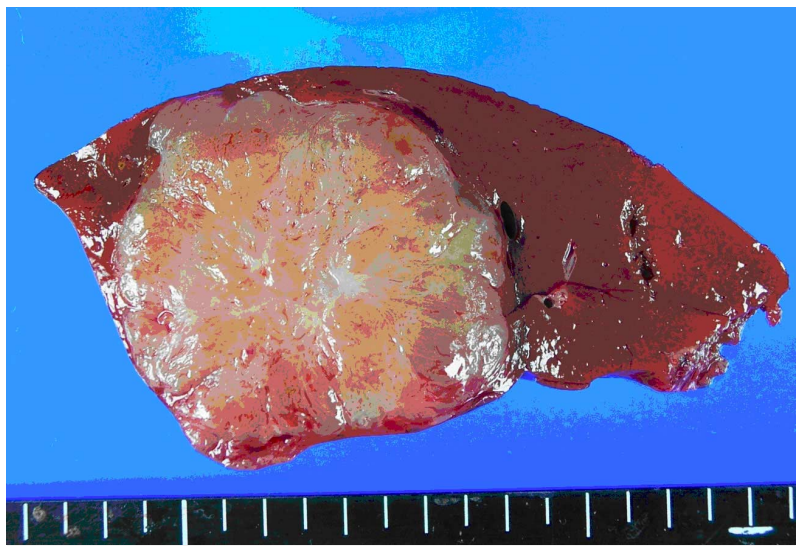

Fig. 4 The cut surface of the tumor is nodular, yellow to tan and a well-defined margin with no capsule.

clear cell cytoplasm. Some of cells exhibited cellular pleomorphism, with hyper chromatic nuclei, but there was no mitotic activity. Immunohistochemically, the smooth muscle cells showed positive reactions for HMB45 (Fig. 6) and actin, but negative for cytokeratin 


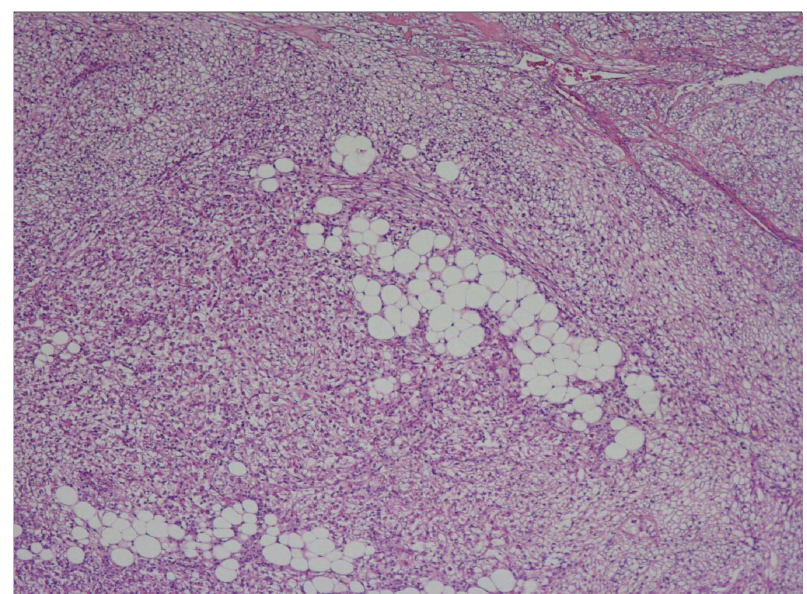

Fig. 5 The tumor surgically removed is composed of irregularly arranged sheets and interlacing bundles of epithelioid smooth muscle cells and a small amount of mature adipose tissue. Thick-walled, medium-sized vessels are also observed as the tumor elements. The epithelioid smooth muscle cells have eosinophilic or clear cell cytoplasm. Some of cells exhibit cellular pleomorphism, with hyper chromatic nuclei, but there is no mitotic activity.

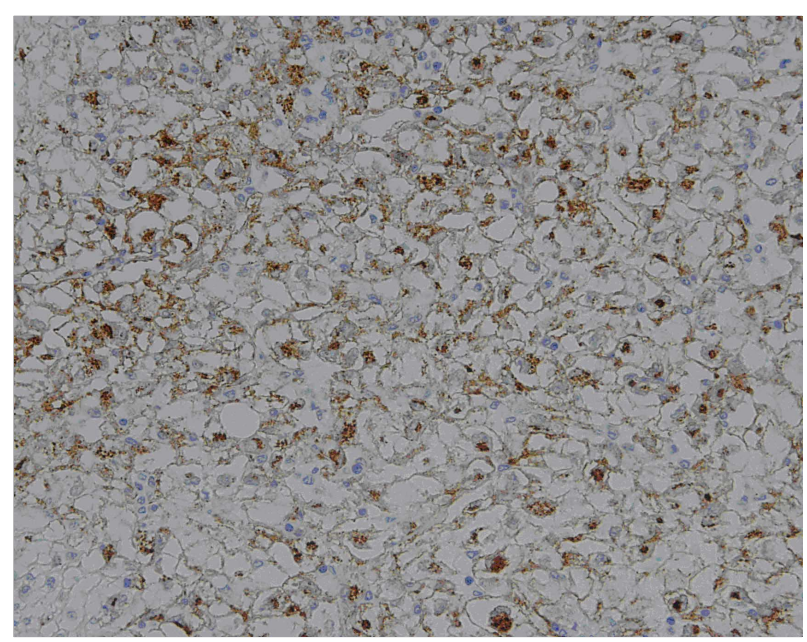

Fig. 6 Immunohistochemically, the smooth muscle cells show positive reactions for HMB45 and actin, but negative for cytokeratin and desmin.

and desmin. Based on these light microscopic and immunohistochemical results, the tumor was diagnosed as AML. The patient is doing well 2 years after surgery.

\section{Discussion}

In this case, we preoperatively diagnosed the tumor as HCC by imaging and biopsy examinations without a concern of AML. Although the combination of US, CT and MRI increases the correct preoperative diagnosis of AML, it remained difficult ${ }^{2}$. Evaluation of the hemodynamic characteristics of a hypervascular tumor might offer useful information to differentiate the tu- mor. One study described the contrast CT images in which many lesions were markedly enhanced with the central vascular opacification in arterial phase ${ }^{6}$. And many lesions remained in enhancement in the portal venous phase. Another study demonstrated some characteristic features of AML, such as fat components within the lesion, hypervascular tumors, and the absence of capsule ${ }^{7}$. In particular the central vessels within the lesions suggested a diagnosis of AML. The feeding vessel can be seen in other hypervascular lesions such as HCC and FNH, but the vessels in those cases usually are located in the periphery of the lesion.

The clinical differential diagnosis was considered as follows, lipoma, hemangioma, FNH and HCC. In this case, the contrast enhanced dynamic CT revealed an enhanced lesion in the arterial phase with washout in the portal phase. AML with a poor fatty component should be considered in the presence of rapid wash-out intravenous contrast injection ${ }^{8}$.

A variety of liver lesions ranging from benign entities to malignant tumors may contain fat ${ }^{10)}$. The benign lesions include focal or geographic fatty change, hepatocellular adenoma, FNH, lipoma, angiomyolipoma. Malignant tumors that can contain fat primarily include HCC, primary and metastatic lipoma. It is difficult to differentially diagnose AML from HCC with fatty metamorphosis. Fat has a characteristic appearance with each of the major cross-sectional imaging modalities. It usually appears hyperechoic at US. Fat is of low attenuation compared with normal liver parenchyma at CT. MRI allows fat suppression and multiphase dynamic scanning. AML shows hyperintensity on fast spin echo T2 weighted images and high signal intensity on T1 weighted images ${ }^{6}$. Our case demonstrated hypointense on T1, hyper intense on T2 and heavy T2 weighted images. On gadolinium enhanced dynamic MRI, the lesion showed enhancement in the early phase with central scar and wash-out of contrast material in the late phase. Because of atypical imaging, the diagnoses remained difficult and differentiation from other hyper vascular tumors was not possible.

Histologically, AML was characterized by an admixture of mature fat cells, blood vessels, and smooth muscle cells (SMC) $)^{9}$. Some features of AML are considered conducive to an erroneous diagnosis of HCC: a varied amount of SMC, hypercellularity, pleomorphism, and moderate mitotic activity. Because of that the pathological diagnosis is difficult, especially on needle biopsy specimens. Nonomura et al. ${ }^{10)}$ performed an immunohistochemical study on nine AML. All tumor components were negative for epithelial membrane antigen and for cytokeratin. Epithelioid SMC were negative for vimentin, desmin and alphasmooth muscle actin. Both the epithelioid and spindle- 
shaped SMC were occasionally positive for S-100 and neuron-specific enolase. All types of SMC in the tumor were strongly positive for HMB45. HMB45 is considered as a monoclonal antibody for melanoma. But positive reaction to purified HMB45 is shown by the other tumors ${ }^{11)}$. HMB45 positive myoid cells in the liver tumor constitute the defining criterion for diagnosis of $\mathrm{AML}^{3)}$.

Although there were many kinds of images and biopsy specimens obtained, we could not obtain correct diagnosis before surgery. When we encountered atypical images of the live tumor, we should inform those findings to the pathologist preoperatively and request the examination of immunohistochemical staining especially for HMB45 on biopsy specimens.

Preoperative diagnosis of AML is still difficult for atypical case even with the comprehensive imaging and needle biopsy. AML needs to be considered as a condition that radiologically mimics HCC. Because AML is considered a benign tumor, surgical intervention should be as limited as possible. To avoid unnecessary hepatectomy, clinical judgment assisted by the immunohistochemical staining for HMB45 on biopsy specimens should be of value when a fat-containing liver tumor remains unconfirmed despite extensive investigation.

\section{References}

1) Sajima S, Kinoshita H, Okuda K, et al. Angiomyolipoma of the liver-a case report and review of 48 cases reported in Japan. Kurume Med J 1999; 46 (2): 127-131.

2) Yang CY, Ho MC, Hu RH, et al. Management of hepatic angiomyolipoma. J Gastrointest Surg 2007; 11 (4): 452 457.

3) Tsui WM, Colombari R, Portmann BC, et al. Hepatic angiomyolipoma: a clinicopathologic study of 30 cases and delineation of unusual morphologic variants. Am J Surg Pathol 1999; 23 (1): 34-48.

4) Yeth $\mathrm{CN}$, Chen MF, hung CF, et al. Angiomyolipoma of the liver. J Surg Onchol 2001; 77 (3): 195-200.

5) Tui WM, Yuen AK, Ma KF, et al. Hepatic angiomyolipomas with a deceptive trabecular pattern and HMB-45 reactivity. Histopathology 1992, 21: 569-573.

6) Ceyla B, Musturay K, Deniz A, et al. Fat-containing lesions of the liver: Cross-sectional image findings with emphasis on MRI. AJR 2005; 184: 1103-1110.

7) Yan F, Zeng M, Zhou K, et al. Hepatic angiomyolipoma: various appearances on two-phase contrast scanning of spiral CT. Eur J Radiol 2002; 41 (1): 12-20.

8) Flor N, Sardanelli F, Serantoni S, et al. Low-fat angiomyolipoma of the liver with contrast-enhanced ultrasound and multidetector computed tomography. Acta Radiol 2006; 47 (6): 543-546.

9) Nonomura A, Mizukami Y, Kadoya M. Angiolipoma of the liver: a collective review. J Gastroenterol 1994; 29 (1): 95105.

10) Nonomura A, Mizukami Y, Takayanagi N, et al. Immunohistochemical study of hepatic angiomyoli-poma. Pathology International 1996; 46: 24-32.

11) Gaffey MJ, Mills SE, Zarbo RJ, et al. Immunohistochemical and ultrastructural evidence of melanogenesis. Am J Surg Pathol 1991; 15: 644-653. 